09,03

\title{
Кинетика излучения поверхностных (би) экситонов в тонких пленках ZnO
}

\author{
(c) И.Х. Акопян ${ }^{1}$, М.Э. Лабзовскаяя ${ }^{1,}$, Б.В. Новиков ${ }^{1}$, V.G. Talalaev' ${ }^{2}$, \\ J.W. Tomm ${ }^{3}$, J. Schilling ${ }^{2}$ \\ ${ }^{1}$ Санкт-Петербургский государственный университет, \\ Санкт-Петербург, Россия \\ ${ }^{2}$ Martin-Luther-University, Centre for Innovation Competence SiLi-nano, \\ Halle, Germany \\ ${ }^{3}$ Max Born Institute for Nonlinear Optics and Short Pulse Spectroscopy, \\ Berlin, Germany \\ ฯ E-mail: xrul@mail.ru
}

(Поступила в Редакцию 10 октября 2018 г.)

\begin{abstract}
Исследована кинетика близкраевой фотолюминесценции (ФЛ) в нанометровых пленках $\mathrm{ZnO}$, полученных методом слоевого наслаивания (ALD). Установлено, что кинетика близкраевой ФЛ в 4-nm пленках в большой степени определяется поверхностными 2D-экситонными (SX) и биэкситонными (SXX) комплексами. Вклад поверхностных биэкситонов оценивается на основе фотостимулированного изменения поверхностного потенциала в пленках $\mathrm{ZnO}$ различной толщины. Обнаружена ультрабыстрая динамика поверхностных биэкситонов в тонких пленках. Показано, что биэкситоны, локализованные вблизи поверхности, имеют самое короткое излучательное время жизни (менее 100 ps) из всех связанных экситонных комплексов, что объясняется большой силой осциллятора.
\end{abstract}

Сотрудники СПбГУ выражают благодарность РФФИ за финансовую поддержку (№ гранта 18-03-00754).

DOI: 10.21883/FTT.2019.03.47247.276

\section{1. Введение}

Ультрабыстрая кинетика излучательных процессов с участием экситонов в широкозонных полупроводниках в последние десятилетия является предметом многочисленных исследований [1]. Возросший интерес к этим исследованиям связан с перспективой создания на базе наноразмерных полупроводниковых систем с большой энергией связи экситона различных УФ-излучательных устройств, способных работать при комнатных температурах и выше. Теоретически предсказанное усиление нелинейных оптических явлений в низкоразмерных системах [2], связанное с увеличением сил осциллятора межзонных оптических переходов, подтверждается экспериментально [3-7]. Наблюдается понижение пороговых значений плотности возбуждения для различных процессов экситон-экситонного взаимодейстия - образования биэкситонов, неупругого экситон-экситонного рассеяния, лазерного эффекта [4-7]. Есть данные [5] об усилении энергии связи экситонов и биэкситонов в низкоразмерных системах по сравнению с энергией в объемных образцах.

Известно, что состояние поверхности $\mathrm{ZnO}$ существенно влияет на его оптические и электрические свойства [8]. Наши предшествующие исследования по влиянию газовой среды [9] и УФ-засветки [10] на спектр интегрированной по времени ФЛ, возбуждаемой $\mathrm{He}-\mathrm{Cd}$-лазером, показали, что адсорбция и десорбция различных газов обратимо меняет спектр ФЛ в экситонной и примесной областях спектра.
В настоящей работе исследования близкраевой люминесценции продолжены с использованием спектроскопии временного разрешения. Времена излучательных процессов в $\mathrm{ZnO}$ изучались многократно [1]. Имеются данные о временах релаксации поляритонов, связанных экситонов, биэкситонов и других процессов. Часто эти данные трудно сопоставлять, так как они получены на объектах, выращенных разными методами и имеющих различную морфологию. В настоящей работе исследуется кинетика ФЛ в образцах с известной морфологией, которая хорошо воспроизводится благодаря технологии атомного наслаивания [11].

\section{2. Эксперимент}

Исследовались пленки $\mathrm{ZnO}$ толщиной 4 и $100 \mathrm{~nm}$. По данным сканирующей электронной микроскопии пленки имели поликристаллическую структуру: 4-х нанометровые пленки состояли из кристаллитов сферической формы диаметром 4-5 nm; пленки толщиной $100 \mathrm{~nm}$ состояли из плотно упакованных слегка вытянутых образований частиц с характерным размером 20-30 nm.

Кинетика фотолюминесценции исследовалась при $T=5 \mathrm{~K}$ на экспериментальной установке с временным разрешением (BР) 10 ps. Возбуждение с длиной волны $260 \mathrm{~nm}$ осуществлялось системой из импульсного титан-сапфирового лазера Tsunami с фундаментальной длиной волны $780 \mathrm{~nm}$, генератора второй гармоники 
$(390 \mathrm{~nm})$ и сумматора частот (SFG) фундаментальной и второй гармоники. $100 \mathrm{fs}$ импульсы задающего лазера следовали с частотой $82 \mathrm{MHz}$ и синхронизировали детектирование ФЛ-стрик-камерой Hamamatsu, оснащенной УФ-катодом S20 и установленной на выходе $300 \mathrm{~mm}$ монохроматора Acton-SP. Спектральное разрешение $0.25 \mathrm{~nm}$ достигалось выбором дифракционной решетки и раскрытием входной щели монохроматора. Для охлаждения образца $\mathrm{ZnO}$ использовался гелиевый криостат замкнутого цикла. Мощность SFG возбуждения максимальной плотности $200 \mathrm{~W} / \mathrm{cm}^{2}$ (в импульce $3 \cdot 10^{12}$ photons $/ \mathrm{cm}^{2}$ ) регулировалась нейтральными фильтрами.

Анализ спектров ФЛ с временным разрешением (ФЛВР) проводился в интервале $10-1200$ ps. Уровень фона извлекался аппаратно. Профили спада интенсивности ФЛ и их спектральное распределение получались интегрированием в окне шириной $0.25 \mathrm{~nm}$, соответствующей инструментальному разрешению. Уровень фона извлекался аппаратно. Подгонка профиля ФЛВР одной экспонентой давала постоянную времени спада ФЛ $(\tau)$ и считалась обоснованной, если величина $\tau$ определялась с точностью не хуже $5 \%$.

\section{3. Результаты и обсуждение}

На рис. 1 представлены спектры излучения пленки $\mathrm{ZnO}$ при различных уровнях возбуждения фемтосекундными импульсами $(\lambda=260 \mathrm{~nm})$ при $5 \mathrm{~K}$. При плотности $2 \mathrm{~W} / \mathrm{cm}^{2}$ наблюдаются две полосы излучения с максимумами при $\lambda=368.8 \mathrm{~nm}$ и $\lambda=374.5 \mathrm{~nm}$. Вид спектра практически не отличается от спектра ФЛ-образца при малом возбуждении $\mathrm{He}-\mathrm{Cd}$-лазером [11].

Следует заметить, что в спектральную область коротковолновой полосы ФЛ при низких температурах попадает излучение, возникающее при нескольких рекомбинационных механизмах - излучательном распаде поверхностных экситонов SX (полоса с максимумом $368.4-368.5 \mathrm{~nm})[12,13]$, экситонов, связанных на ионизованных $\left(I_{2}, I_{3}\right)$ и нейтральных $\left(I_{4}-I_{10}\right)$ донорах $(368.1-370.0 \mathrm{~nm})[14]$, излучательного распада биэкситона XX (368.8-368.9 nm) [4-6,15,16]. В тонких пленках и вискерах в близкраевой области спектра преобладает излучение поверхностных экситонов.

Характерное для спектра ФЛ $\mathrm{ZnO}$ проявление полос разной природы в узкой спектральной области спектра имеет место и в длинноволновой полосе, куда попадают полосы, обусловленные переходами свободный электрон зоны проводимости-акцептор (374.5 nm) [17], излучением донорно-акцепторных пар при переходах локализованного электрона на мелкий акцептор $(374.2 \mathrm{~nm})$ [18], полосы двухэлектронных сателлитов связанных экситонов $(374-375 \mathrm{~nm})$ [14], неупругого экситон-экситонного рассеяния $(373.9 \mathrm{~nm})[6,16]$ и первого LO-фононного повторения свободного экситона $(375.0 \mathrm{~nm})$ [19].
При интерпретации полос и измерении времени релаксации в импульсном режиме нами учитывалась разная вероятность проявления того или иного механизма при различных уровнях возбуждения. Очевидно, при относительно низких уровнях возбуждения при $5 \mathrm{~K}$ излучательные переходы связаны в основном с экситонами и дефектами. При повышении плотности возбуждения следует ожидать доминирующего вклада эффектов экситон-экситонного взаимодействия излучения биэкситонов в коротковолновой полосе и экситон-экситонного рассеяния в длинноволновой.

При увеличении уровня возбуждения в спектре ФЛ-пленки (рис. 1) выделяется и растет линия при $\lambda=369.3 \mathrm{~nm}$ (на рис. 1 это линия SXX). При плотности возбуждения $200 \mathrm{~W} / \mathrm{cm}^{2}$ она превосходит по интенсивности доминирующие при меньших интенсивностях возбуждения линии связанных экситонов.

Было обнаружено также, что предварительное облучение пленки фемтосекундными импульсами в течение нескольких минут приводит к дополнительному росту относительной интенсивности линии SXX. Рис. 2 иллюстрирует эффект предварительного облучения пленки на спектры ФЛ при разных уровнях возбуждения $63 \mathrm{~W} / \mathrm{cm}^{2}$ и $200 \mathrm{~W} / \mathrm{cm}^{2}$ (рис. $2, a$ и $b$ соответственно). При одинаковой продолжительности облучения конечный результат зависит от уровня возбуждения. Таким образом, наблюдения показывают, что интенсивность линии SXX определяется как плотностью возбуждения, так и временем облучения, то есть зависит от полученной дозы облучения.

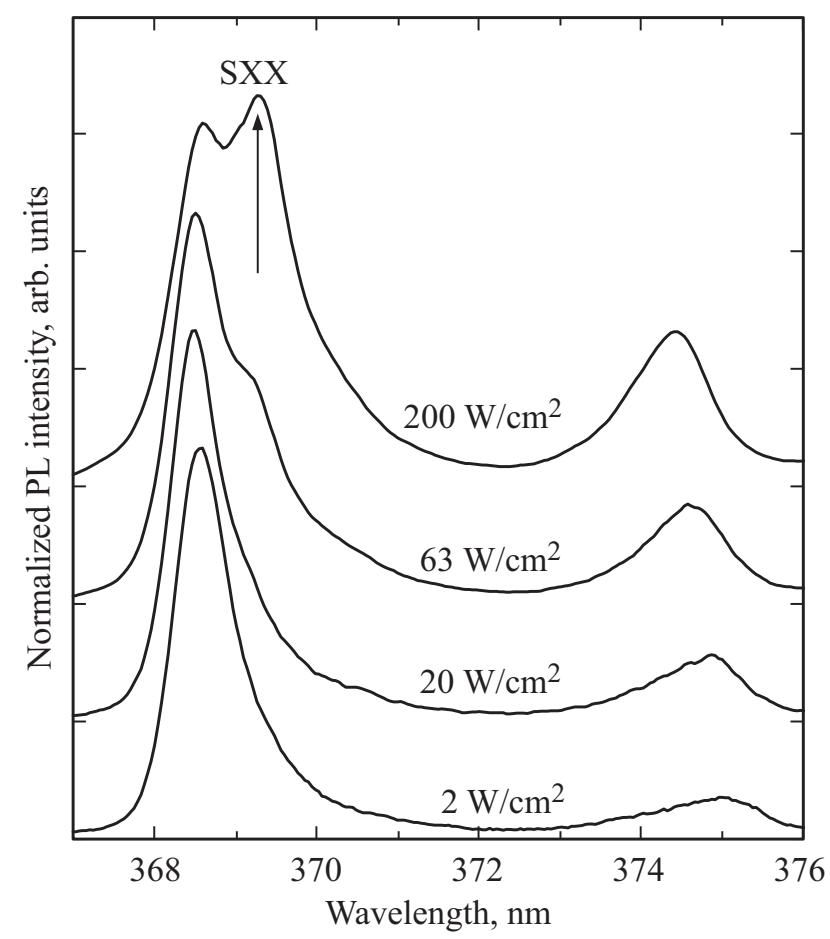

Рис. 1. Спектры ФЛ-пленки $\mathrm{ZnO}$ при $T=5 \mathrm{~K}$ и различных плотностях мощности возбуждения. 
Времена релаксации полос ФЛ пленки толщиной $4 \mathrm{~nm}$ при $T=5 \mathrm{~K}$ в зависимости от плотности мощности возбуждения $(P)$

\begin{tabular}{|c|c|c|c|c|c|}
\hline \multirow{2}{*}{$\begin{array}{c}\text { Полоса } \\
\text { ФЛ }\end{array}$} & \multirow{2}{*}{$\begin{array}{c}\lambda, \mathrm{nm} \text { при } \\
P=200 \mathrm{~W} / \mathrm{cm}^{2}\end{array}$} & \multicolumn{4}{|c|}{ Время спада ФЛ $\tau$,ps в максимуме полосы } \\
\hline & & $P=200 \mathrm{~W} / \mathrm{cm}^{2}$ & $P=63 \mathrm{~W} / \mathrm{cm}^{2}$ & $P=20 \mathrm{~W} / \mathrm{cm}^{2}$ & $P=2 \mathrm{~W} / \mathrm{cm}^{2}$ \\
\hline SX & 368.5 & 135 & 105 & 90 & 85 \\
\hline DX & 368.9 & 130 & 110 & 95 & 90 \\
\hline SXX, abs. min & 369.3 & 100 & 100 & 95 & 95 \\
\hline $\mathrm{AX}$ & 370.1 & 110 & 105 & 100 & 100 \\
\hline$a$ & 373.9 & 430 & 380 & 350 & 260 \\
\hline$b$ & 374.4 & 650 & 600 & 450 & 350 \\
\hline$c$, abs. max & 374.7 & 1020 & 1030 & 900 & 610 \\
\hline
\end{tabular}

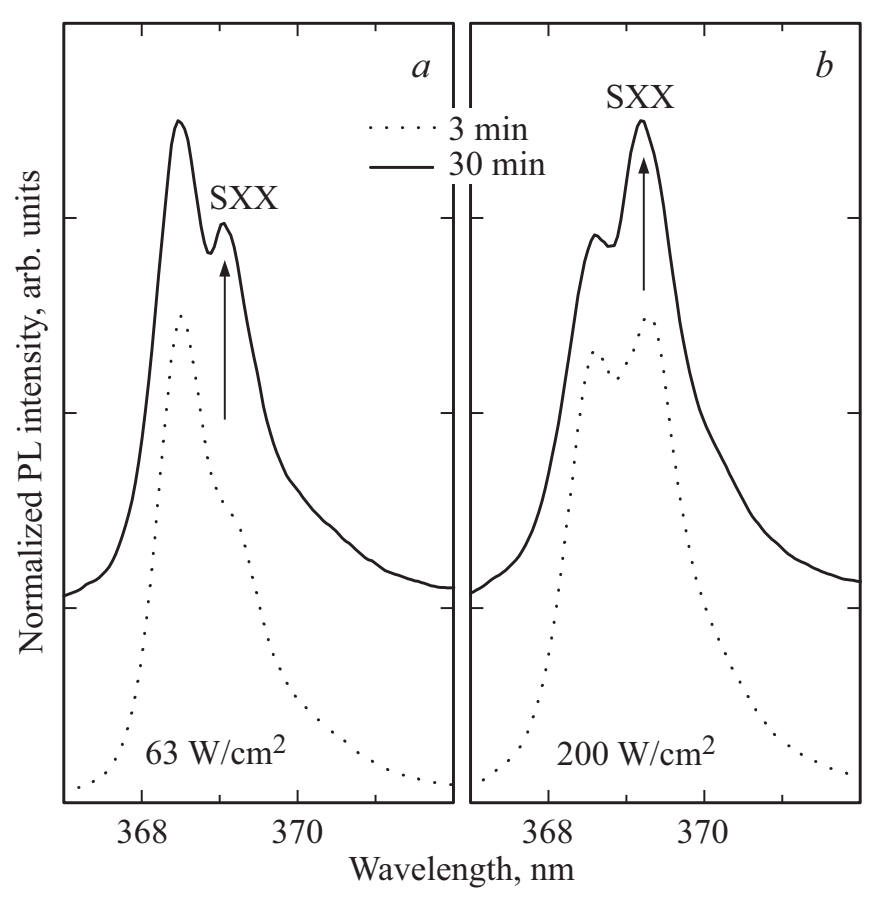

Рис. 2. Спектры ФЛ-пленки $\mathrm{ZnO}$ при $T=5 \mathrm{~K}$ и различных плотностях и длительностях предварительного облучения.

На рис. 3 представлен скриншот стрик-камеры $(a)$, показан спектр интегрированной по времени ФЛ-пленки $\mathrm{ZnO}$ толщиной $4 \mathrm{~nm}$ при $200 \mathrm{~W} / \mathrm{cm}^{2}$ с разложением полос на лоренцианы $(b)$ и приведены полученные зависимости времен спада ФЛ $(\tau)$ при различных уровнях возбуждения для двух спектральных областей $(c$ и $d)$.

В таблице приведены времена спада в зависимости от интенсивности возбуждения для основных максимумов ФЛ. Отметим, что для всех максимумов наблюдается рост времен с увеличением плотности возбуждения, что может быть связано с насыщением центров безизлучательной аннигиляции носителей заряда и экситонов.

Как видно из рис. 3,c, в коротковолновой области ход времени спада обнаруживает сложную структуру, существенно зависящую от уровня возбуждения. При $2 \mathrm{~W} / \mathrm{cm}^{2}$ наблюдается четкий минимум при $\lambda=368.4 \mathrm{~nm}$. С ростом возбуждения его глубина умень- шается, а при $200 \mathrm{~W} / \mathrm{cm}^{2}$ он мало заметен. Мы считаем, что минимум при $368.4 \mathrm{~nm}$ обусловлен излучением поверхностных экситонов (SX). Известно, что излучательное время поверхностных экситонов меньше времени свободных и связанных экситонов [13]. С ростом интенсивности возбуждения это время увеличивается и минимум сглаживается.

При $63 \mathrm{~W} / \mathrm{cm}^{2}$ начинает формироваться минимум при $\lambda=369.7 \mathrm{~nm}$, который при $200 \mathrm{~W} / \mathrm{cm}^{2}$ становится основной структурой. Следует заметить, что время спада $(\tau)$ в этом минимуме практически не зависит от уровня возбуждения, а его образование можно рассматривать как рост времени с коротковолновой и длинноволновой сторон от минимума. Минимум при $369.7 \mathrm{~nm}$ мы связываем с излучением поверхностных биэкситонов (SXX). Его присутствие четко коррелирует с возникновением максимума ФЛ в указанной спектральной области. В пользу биэкситонной природы указанной полосы свидетельствуют ее возникновение при высокой плотности возбуждения, сверхлинейная зависимость интенсивности от плотности возбуждения, спектральное положение линии. В этом спектральном интервале в спектре ФЛ ранее наблюдалась полоса, приписываемая в [20,21] биэкситону, локализованному на доноре. Принадлежность полосы SXX локализованному состоянию подтверждается обнаруженной в настоящей работе зависимостью ее интенсивности от дозы облучения. Полученные малые времена спада ФЛ согласуются с результатами теоретических и экспериментальных [22-24] работ.

На рис. 4 показан ход времени спала ФЛ для пленок разной толщины в спектральной области коротковолновой полосы. Видно, что поверхностные особенности (SX и SXX) более отчетливо наблюдаются в тонкой пленке.

На рис. 3, $d$ показаны спектральные зависимости времени спада ФЛ в области длинноволновой полосы при $374.5 \mathrm{~nm}$ в спектре интегрированной по времени ФЛ для разных уровней возбуждения. Разложение на лоренцианы показывает, что в соответствии с вышесказанным в спектральной области полосы могут проявляться три структуры разной природы (полосы $a, b, c$ на рис. $3, d$ ). Ранее нами обнаружено, что при росте интенсивности возбуждения максимум всей полосы в интегрированной по времени ФЛ смещается в коротковолновую сторону 


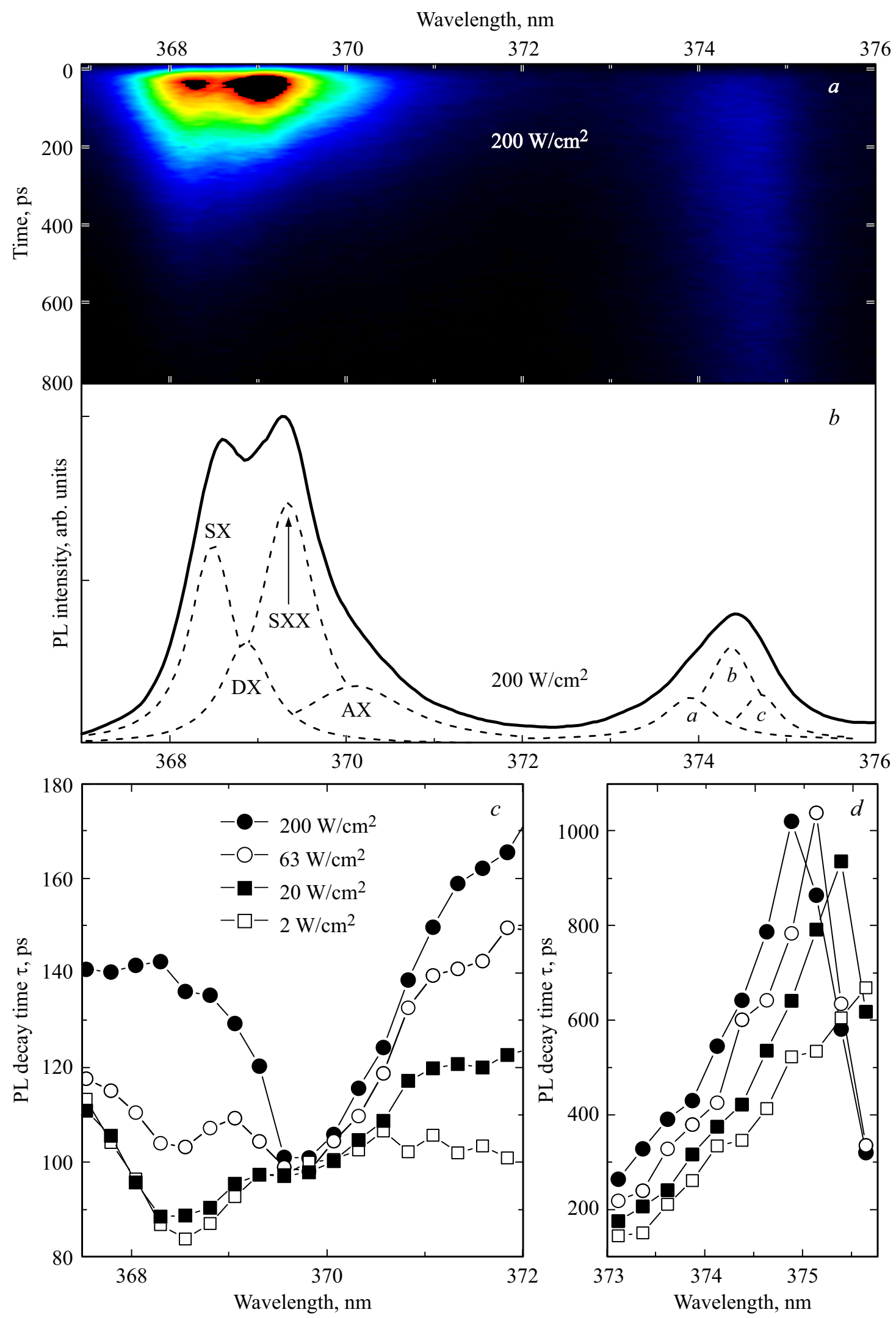

Рис. 3. Скриншот стрик-камеры (a), спектр интегрированной по времени ФЛ-пленки толщиной $4 \mathrm{~nm}$ (сплошная линия) с разложением полос на лоренцианы (пунктирные линии) $(b)$ в зависимости от времени спада ФЛ при различных уровнях возбуждения для двух спектральных областей $(c$ и $d)$. 


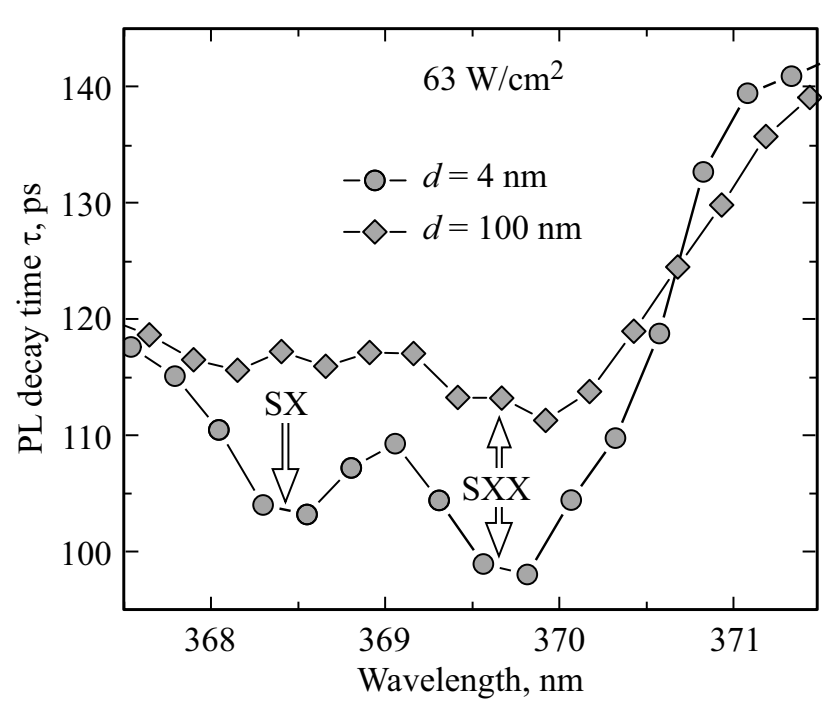

Pис. 4. Времена спада ФЛ-пленок $\mathrm{ZnO}$ разной толщиныв при $5 \mathrm{~K}$ в спектральном интервале $368.0-372.0 \mathrm{~nm}$.

(рис. 1). Из рис. 3, $d$ видно, что время спада во всей полосе $(373-376 \mathrm{~nm})$ увеличивается в длинноволновую сторону, при этом в области $b$-полосы этот рост заметно ускоряется. Обнаруженное поведение отвечает свойствам ФЛ донорно-акцепторных пар, когда переход электрона на акцепторный уровень происходит из локализованного состояния. В $\mathrm{ZnO}$ полосы излучения ДАП (донорно-акцепторные пары), как правило, наблюдаются в более длинноволновой области (385 nm и далее) и в основном в легированных образцах. О полосе излучения ДАП в $\mathrm{ZnO}$ с мелким акцептором сообщалось в [19] для вискеров. Заметим, что при малом возбуждении $2 \mathrm{~W} / \mathrm{cm}^{2}$, соответствующем максимальным в нашем случае расстояниям в парах, скорость роста времени постоянна во всей полосе. Это соответствует ожидаемому для ДАП уменьшению интенсивности при больших расстояниях между носителями в парах. Мы полагаем, что основной вклад в излучение $b$-полосы вносят донорно-акцепторные пары.

\section{4. Заключение}

Исследована кинетика близкраевых излучательных процессов при $T=5 \mathrm{~K}$ в нанокристалличеких пленках $\mathrm{ZnO}$ толщиной 4 и $100 \mathrm{~nm}$. В результате проведенных исследований измерены времена спада ФЛ в максимумах полос излучения в зависимости от плотности возбуждения в диапазоне $2-200 \mathrm{~W} / \mathrm{cm}^{2}$.

Показано, что для разных излучательных процессов времена релаксации меняются в зависимости от величины плотности возбуждения в интервале от десятков до сотен ps. Проведен анализ спектральной зависимости времени спада ФЛ для разных уровней возбуждения ФЛ. Совокупность экспериментальных данных позволила установить, что при низких уровнях возбуждения в исследованных пленках $\mathrm{ZnO}$ эффективным рекомбинационным каналом является излучение поверхностных экситонов (SX), тогда как при высоких уровнях возбуждения более эффективно излучение локализованных биэкситонов (SXX).

Показано, что экситоны и биэкситоны, локализованные вблизи поверхности, имеют самое короткое излучательное время жизни из всех связанных экситонных комплексов, что объясняется большой силой осциллятора, обеспеченной значительным перекрыванием волновых функций электронов и дырок в двумерном случае. Обнаружено, что поверхностные особенности более отчетливо проявляются в $4 \mathrm{~nm}$ пленке по сравнению с соответствующими эффектами в пленке $100 \mathrm{~nm}$.

\section{Список литературы}

[1] S.F. Klingshirn, B.K. Meyer, A. Waag, A. Hoffmann, J. Geurts. Zinc Oxide, Springer (2010).

[2] Y. Kayanuma. Phys. Rev. B 38,9797 (1998).

[3] S. Wu, L. Cheng, Q. Wang. Mater. Res. Express 4, 8, 085017 (2017).

[4] H.J. Ko, Y.E. Chen, T. Yao, K. Miyajima, A. Yamamoto, T. Goto. Appl. Phys. Lett. 77, 537 (2000).

[5] H.D. Sun, T. Makino, K. Tamura, Y. Segava, M. Kavasaki, A. Ohtomo, K. Tamura, H. Koinuma. Phys. Status Solidi B 229, 867 (2002).

[6] C.H. Chia, Y.J. Lai, T.C. Han, J.W. Chiou, Y.M. Hu, W.C. Chou. Appl. Phys. Lett. 96, 081903 (2010).

[7] D. Vanmaekelbergh, Lambert K. van Vugt. Nanoscale 3, 2783 (2011).

[8] M. Ghost, R.S. Ningthoujam, R.K. Vatsa, D. Das, V. Nataraju, S. Gadkari, S.K. Gupta, D. Bahadur. J. Appl.Phys. 110, 054309 (2011).

[9] И.Х. Акопян, М.Э. Лабзовская, А.А. Лисаченко, Б.В. Новиков, А.Ю. Серов, В.В. Титов, Н.Г. Философов. ФТТ 58, 1709 (2016).

[10] И.Х. Акопян, М.Э. Лабзовская, Б.В. Новиков, А.А. Лисаченко, А.Ю. Серов, Н.Г. Философов. ФТТ 60, 346 (2018).

[11] M.E. Labzovskaya, I.Kh. Akopyan, B.V. Novikov, A.Yu. Serov, N.G. Filosofov, L.L. Basov, V.E. Drozd, A.A. Lisachenko. Phys. Procedia 76, 37 (2015).

[12] V.V. Travnikov, A. Freiberg, S.F. Savikhin. J. Luminescence 47, 107 (1990).

[13] L. Wishmeier, T. Voss, I. Ruckmann, J. Gutowski, A.C. Mofor, A. Bakin, A. Waag. Phys. Rev. B 74, 195333 (2006).

[14] B.K. Meyer, H. Alves, D.M. Hofmann, W. Kriegsels, D. Forster, F. Bertram, J. Christen, A. Hofmann, M. Strassburg, M. Dworzak, U. Haboeck. Phys. Status Solidi B 241 (2004).

[15] Fang-Li Yen, Yen-Cheng Lu, Cheng-Yen Chen, Hsiang-Chen Wang, C.C. Yang, Bao-ping Zhang, Y. Segawa. Appl. Phys. Lett. 87, 072103 (2005).

[16] H. Murotani, D. Akase, Y. Yamada, T. Matsumoto, D. Nakamura, T. Okada. Appl. Energy Eng. 1011 (2010).

[17] M. Schirra, R. Schneider, A. Reiser, G.M. Prinz, M. Feneberg, J. Biskupek, U. Kaiser, C.E. Krill, K. Thonke, R. Sauer. Phys. Rev. B 77, 125215 (2008).

[18] S.H. Eom, Y.-M. Yu, Y.D. Choi, C.-S. Kim. J. Cryst. Growth 284, 166 (2005). 
[19] D. Tainoff, B. Masenelli, P. Melinon, A. Belsky, G. Ledoux, D. Amans, C. Dujardin, N. Fedorov, P. Martin. Phys. Rev. B 81, 115304 (2010); P. Corfdir, M. Abid, A. Mouti, Pierre A. Stadelmann, E. Papa, J-P. Ansermet, J-D. Ganiére, B. Deveaud-Plédran. Nanotechnology 22, 285710, (2011).

[20] A. Yamamoto, K. Miyamia, T. Goto, H.J. Ko, N. Yao. Phys. Status Solidi B 229, 871 (2002).

[21] Fang-Li Yen, Yen-Cheng Lu, Cheng-Yen Chen, Hsiang-Chen Wang, C.C. Yang, Bao-Ping Zhang, Y. Segawa. Appl. Phys. Lett. 87, 072103 (2005).

[22] D.S. Citrin. Phys. Rev. B 50, 23 (1994). (1999).

[23] M. Nakaeama, H. Ichida, H. Nishimura. J. Phys.: Condens. Matter 11, 7653 (1999).

[24] P. Corfdir, M.A. Anas Mouti, P.A. Stadelmann, E. Papa, JP. Ansermet, J-D. Ganiére, B. Deveaud-Plédran. Nanotechnology 22, 285710 (2011).

Редактор К.В. Емиев 